\title{
Abdominal Wall Endometrioma: Ultrasonographic Features and Correlation with Clinical Findings
}

\author{
Aynur Solak¹, Berhan Genç ${ }^{1}$, Seyhan Yalaz², Neslin Şahin¹, Taylan Özgür Sezer ${ }^{3}$, Illhami Solak ${ }^{3}$ \\ ${ }^{1}$ Department of Radiology, Şifa University Faculty of Medicine, İzmir, Turkey \\ ${ }^{2}$ Department of General Surgery, Şifa University Faculty of Medicine, Izmir, Turkey \\ ${ }^{3}$ Department of General Surgery, Ege University Faculty of Medicine, Izmir, Turkey
}

\section{ABSTRACT}

Background: The diagnosis of abdominal wall endometrioma (AWE) is often confused with other surgical conditions. Certain factors relating to knowledge of the clinical history of the disease make correct diagnosis and treatment difficult.

Aims: To present the clinical findings and ultrasonographic (US) features of AWE with special emphasis on size-related features.

Study Design: This study reviewed abdominal wall endometriomas during a 2-year period in the Radiology Department of Sifa University Hospital, lzmir.

Methods: Eleven women (mean age 32.6 years) with 12 scar endometriomas (mean diameter $29.2 \mathrm{~mm}$ ) were consecutively evaluated by US and Colour Doppler examination (CDUS) prior to surgery. Lesions were grouped into large $(\geq 3 \mathrm{~cm})$ and small nodules. Vascularisation was classified as location (central, peripheral and mixed) and severity (absent, moderately vascular and hypervascular). In each patient, the nature of pain (absent, cyclic: associated with menstruation and continuous), historical and clinical data were documented. Four patients underwent Magnetic Resonance Imaging and their findings were presented. Fisher's exact test, $\chi^{2}$ test for categorical data and the unpaired T-test for continuous variables were used for statistical analysis.

Results: In all the women, US of the AWE showed the presence of a solid hypoechoic mass (less echogenic than the surrounding hyperechoic fat) within the abdominal wall. There was a significant correlation between AWE sizes with repeated caesareans and the mean time between the last operation and admission to hospital $(p<0.05)$. Large endometriomas showed increased central vascularity $(p<0.05)$. Cyclic pain was more frequent in small lesions, whereas continuous pain was more commonly found in patients with larger lesions $(p<0.05)$.

Conclusion: AWE is often misdiagnosed clinically because endometriosis may occur years after the caesarean section, the pain is often non-cyclic in nature, and there is not always a palpable tender mass. The sonographic and Doppler findings, along with proper correlation with clinical data, may substantially contribute to the correct diagnosis of endometrioma.

Key Words: Abdominal wall endometriosis, ultrasonography, cyclic pain

Received: 13.07.2012

Accepted: 17.10.2012

\section{Introduction}

Endometriosis is a term used to describe the presence and growth of functional endometrial tissue outside the uterus. Endometrioma is a well circumscribed mass of endometriosis. It is clinically difficult to differentiate from suture granuloma, abscess, lipoma, haematoma, sebaceous cyst, inguinal hernia, incisional hernia, desmoid tumour or primary and metastatic cancer. It is also difficult to diagnose radiologically, and usually mimics a malignant soft tissue tumour (1-3).

The purpose of our study was to present the clinical findings and ultrasonographic (US) features of abdominal wall endometriosis (AWE) with Magnetic Resonance (MR) findings. Qualitative and quantitative Colour Doppler Ultrasonographic (CDUS) findings are investigated in the lesions grouped according to their sizes. The value of combined assessment using clinical and detailed radiological findings to solve this diagnostic dilemma was discussed and a literature review was performed.

\section{Material and Methods}

\section{Patients}

This study was approved by the institutional review board. All patients previously consented to the use of their medical records for the purpose of study. During the past two years, thirteen patients with AWE were operated upon in our hospital. Their medical and radiological records were found from the picture archiving and communication system (PACS), the hospital information system and the radiology information system (HIS/RIS integrated PACS, Siemens, Erlangen, Germany). Two patients were excluded because of poor-quality images. Eleven women (mean age 32.6 years, range 26-43 years) with 12 scar endometriomas were consecutively seen at the Ultrasound Unit between June 2010 and April 2012. In each patient, complaints (absence or presence of pain in the nodule, cyclic pain that accompanied menstruation or continuous pain), historical (number of caesarean sections, mean time from both last caesarean section or operation and onset 
of symptoms before admission, known pelvic endometriotic foci) and clinical (physical examination at admission) data were documented. Diagnostic procedures (excisional biopsy or US guided Fine Needle Aspiration Biopsy, FNAB) were not performed on the lesions for pre-operative diagnosis. All patients underwent surgery at our institution with a complete resection of the mass and histological examination of the specimen.

\section{Imaging Techniques}

CDUS examination was performed in all patients using 3.5and $5.0-\mathrm{MHz}$ convex-array and 7.5-MHz linear-array transducers (Sonoline Antares, Siemens Medical Solutions, Erlargen, Germany). CDUS was used to evaluate the vascularity of lesions. The following quantitative and qualitative CDUS parameters were detected:

1. Qualitative CDUS of lesions was graded as the presence or absence (avascular, moderately vascular or hypervascular) and classified as the location (peripheral and central) of vascularity in the lesion. The grade of vascularisation was based on the number of vessels: In avascular lesions, no vessel was determined; lesions were moderately vascularised when one or two vascular pedicles were detected; and tumours were highly vascularised when more than two vascular pedicles were seen. On the basis of the distribution of tumour vessels, three patterns of vascularisation were described: a peripheral pattern was a pattern with vessels around the mass periphery and no evidence of branching inside the lesion; a central pattern was a pattern that showed vessels only inside the lesion; and a mixedramifying pattern was a pattern with a deranged distribution of peripheral and intralesional-central vessels.

2. Quantitative measurement of resistance index (RI) was performed in feeding arterial structures. The RI was calculated with the following equation: RI equals (systolic peak velocity minus end-diastolic peak velocity) divided by systolic peak velocity. This was defined for each lesional artery and an average measurement of two waves was made for each artery. The maximum RI value for each lesion was considered in the following analysis.

In addition to US, five patients also underwent MRI on a 1.5-T scanner (Magnetom Espree, Siemens, Germany); those examinations included spin-echo T1-, fast spin-echo fat-saturated T2-, and fast spin-echo fat-saturated contrast-enhanced (intravenous, $0.1 \mathrm{mmol} / \mathrm{kg}$ gadolinium) T1-weighted sequences. All scans were performed in the supine position.

\section{Imaging Analysis}

All ultrasonographic examinations were interpreted by two radiologists working in consensus (AS and BG). Data collection included lesion site, size (largest of three orthogonal dimensions), morphology, echogenicity with respect to the predominant adjacent tissues, and qualitative and quantitative assessment of intralesional blood flow with CDUS. For the purpose of comparison, the lesions were divided into "large nodule" and "small nodule" groups. A large nodule was considered if the widest lesion diameter was equal to or greater than $30 \mathrm{~mm}$. The location, size, signal intensity of the lesions were evaluated on routine-conventional MRI scans. All MR images were re-evaluated on a PACS workstation monitor (Leonardo console software 19A version, Siemens Erlangen Germany).

\section{Histopathological Diagnosis}

A diagnosis of AWE was made histologically when endometrial glands and stroma were found within the soft tissues and muscles of the abdominal wall. They were circumscribed by an inflammatory process, haemosiderin-laden macrophages and fibrosis.

\section{Statistical Analysis}

All clinical and ultrasound information was entered into a dedicated Microsoft Excel file (Microsoft Corp, USA) that was used for statistical analysis. This analysis was performed with SPSS base 21 for windows. Fisher's exact test and $\chi 2$ test for categorical data and the unpaired t-test for continuous variables were used. $p$ values $<0.05$ were considered statistically significant.

\section{Results}

The patients referred to the radiology department were primarily evaluated by the outpatient gynaecologic clinic $(n=5)$, the outpatient surgical clinic $(n=4)$ or a general practitioner $(n=2)$. The presumptive clinical diagnosis before radiologic consultation was abdominal wall endometriosis $(n=4)$, suture granuloma $(n=3)$, appendicitis $(n=2)$, or haematoma $(n=2)$. The clinical characteristics of the 11 women (mean age 32.6 years; range $26-43$ years) constituting the study population are given in Table 1 . All patients had previously delivered a child by caesarean section (mean section operation: 1.8 per patient, min: 1, max: 4). One patient had a known ovarian endometrioma.

The average time between last section and admission to our clinic was 33.2 months (range 19-79 months) and the average time of symptoms before admission was 22.7 months (range 3-45 months). The clinical and demographic features were classified according to lesion size, and these findings were summarised in the same table. Patients with lesion sizes larger than $3 \mathrm{~cm}$ had a longer mean time interval and the longer duration of symptoms before hospital admission when compared to the patients with smaller lesions (34.3 months vs. 12.1 months, $\mathrm{p}<0.05)$. Patients with lesion sizes larger than $3 \mathrm{~cm}$ had a longer mean time interval between the last operation and admission to our clinic, and those with a longer mean time of symptoms before hospital admission also had longer intervals (34.3 months vs. 12.1 months, $\mathrm{p}<0.05$ ) when compared to patients with smaller lesions. The 12 endometriomas had a mean diameter of $29.2 \mathrm{~mm}$ (range 13-58 $\mathrm{mm})$; four patients (36.3\%) had large endometriomas $(\geq 30$ $\mathrm{mm}$ ) with a mean lesion diameter of $41.3 \pm 9.02 \mathrm{~mm}$ (range $30-60 \mathrm{~mm}$ ). Seven women (63.7\%) had small endometriomas with a mean lesion size of 19.2 $\pm 5.18 \mathrm{~mm}$ (range 9-27 mm). Four nodules (33.3\%) were located between the subcutaneous rectus muscle sheath; in three cases (25\%) both the 
subcutaneous and muscular plane were invaded; three lesions $(25 \%)$ were purely subcutaneous and two more nodules (16.7\%) were entrapped in the muscular layer of the abdominal wall. AWEs did not show a preferential distribution along the incision scar: 5 nodules were located on the right, 5 on the left inguinal region and 2 on the midline.

In all women, ultrasound examination of the AWE showed the presence of a solid hypoechoic mass (hypoechogenic compared to the surrounding fat tissue) within the abdominal wall (Figure 1). The echotexture was inhomogeneous in
6 of $12(50 \%)$ cases owing to the presence of inner hyperechoic fibrotic spots, short white cords or small cystic foci (Figure 1a). Nine patients had a single nodule, one patient had two nodules (one large nodule associated with a small lesion) and one patient had multiple adjacent millimetric cysts (large nodule was found surrounded by small chocolate cysts) (Figure 2). There were mixed-branching hypervascular flow patterns in four patients with a lesion size of more than $3 \mathrm{~cm}$ (Figure $1 \mathrm{~b}, \mathrm{c}$ ). In the remaining 8 nodules (66.6\%), all of which were less than $3 \mathrm{~cm}$, peripheral vascu-

Table 1. Table shows clinical data, ultrasonographic findings and statistical results in the study group

\begin{tabular}{|c|c|c|c|}
\hline & L-AWE & S-AWE & $\mathbf{p}$ \\
\hline Mean age (year, range) & $31.6(28-43)$ & $29.3(26-38)$ & NS \\
\hline Number of caesarean sections per patient & $2.5(2-4)$ & $1.3(1-2)$ & $<0.05$ \\
\hline Time since last caesarean delivery (months) & $64.0 \pm 14.5$ & $31.2 \pm 11.3$ & $<0.05$ \\
\hline Onset of symptoms (months before admission) & $34.3 \pm 11.9$ & $12.1 \pm 9.6$ & $<0.05$ \\
\hline Known pelvic endometriotic focus & 0 & 1 & NS \\
\hline Continuous pain & 4 & 0 & $<0.05$ \\
\hline Cyclic pain (pain is associated with the menstrual cycle) & 0 & 5 & $<0.05$ \\
\hline Lesion size $\mathrm{mm}( \pm \mathrm{SD})$ & $41.3 \pm 9.02$ & $19.2 \pm 5.18$ & \\
\hline Resistive Index (mean \pm SD) & $0.76 \pm 0.11$ & $0.69 \pm 0.16$ & NS \\
\hline Central vascularisation & 4 & 0 & $<0.05$ \\
\hline Peripheral vascularisation & 4 & 6 & NS \\
\hline
\end{tabular}
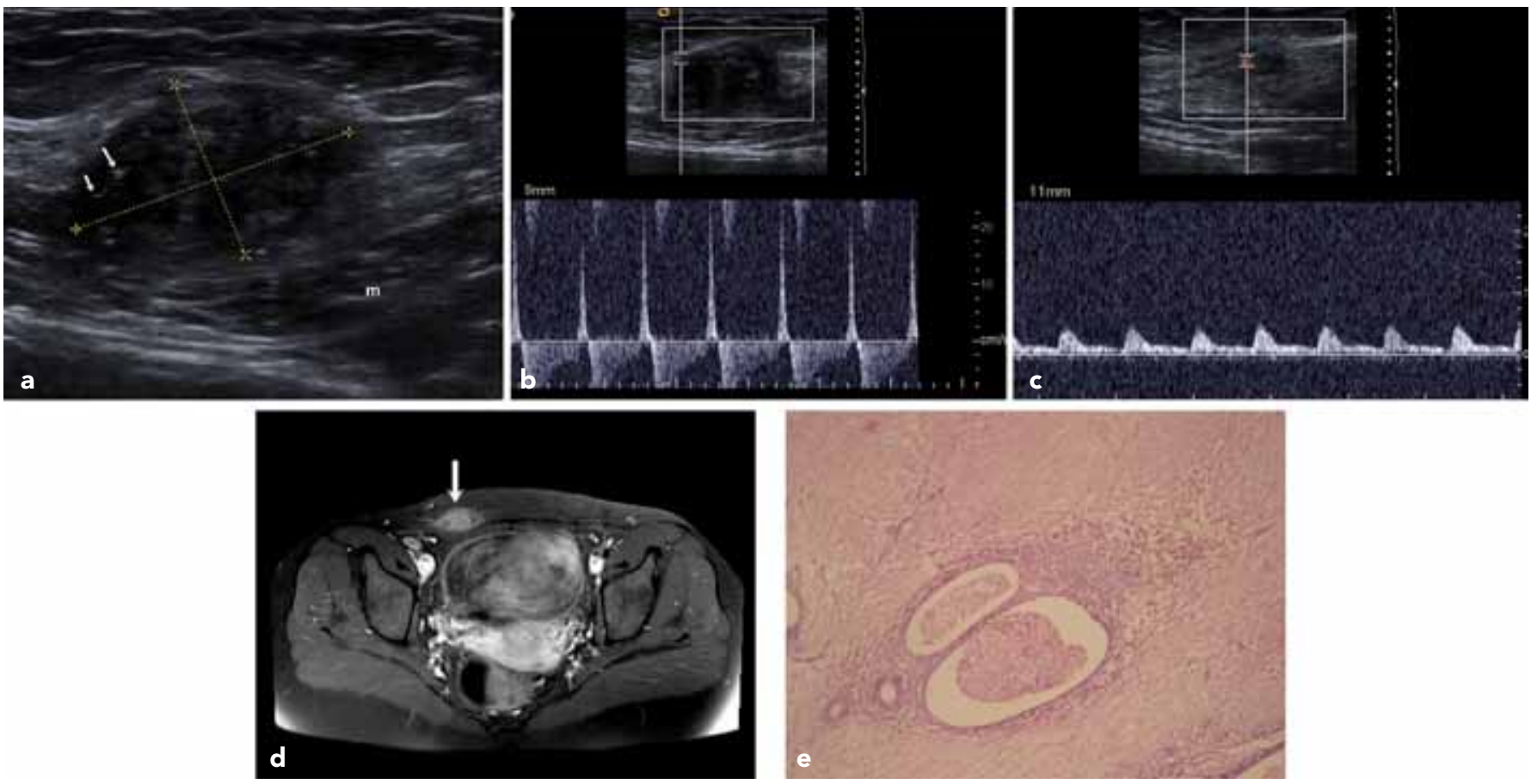

Figure 1. a-e. A 34 year-old woman with 6-month of cyclic pain about two years after a caesarean delivery. Ultrasound examination (a) reveals a well-circumscribed heterogeneous, hypoechoic with fibrotic spots (white arrows), solid mass, 33 x 17 millimetres in size ( $\mathrm{m}$ : rectus muscle). Doppler ultrasound demonstrates high resistance arterial flow (resistive index $>0.7$ ) in the periphery (b) and low resistance flow in the centre (c) of the lesion. Axial T1-weighted fat-saturated spin-echo MR image (d) after injection of contrast medium shows strong enhancement of lesion (arrow). Histological examination (HaematoxylinEosin $\times 40$ ) (e) shows several endometriotic foci scattered in a dense fibrous tissue and muscle fibres. The foci are composed of both endometrial stroma and partially dilated glands in proliferative phase 

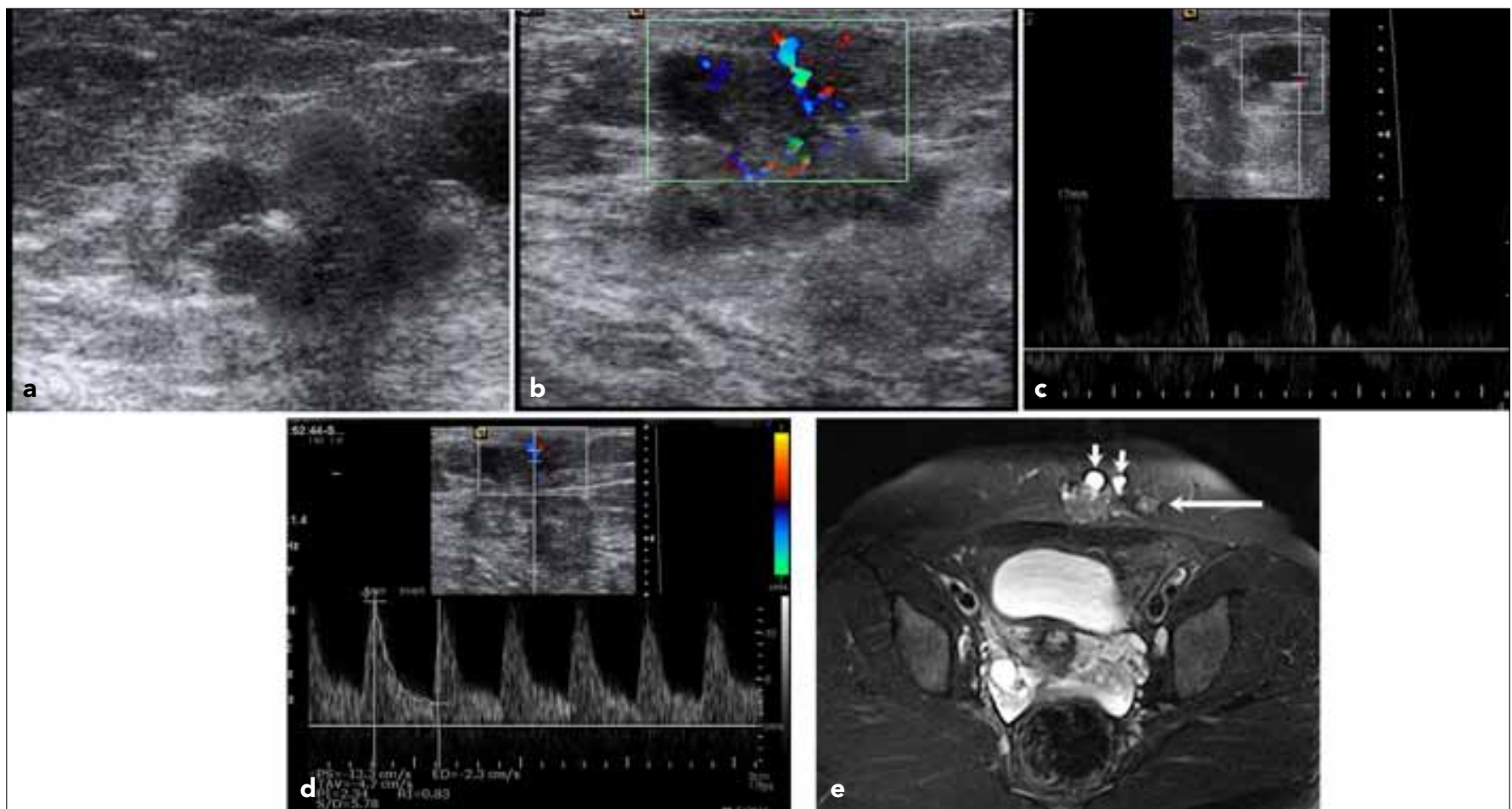

Figure 2. a-e. A 36 year-old woman with a slowly growing painful mass. She has a history of three caesarean sections. Ultrasound (a) shows a $2 \times 3.5 \mathrm{~cm}$ nodule with hypoechoic content and blurred outer margins. Note that multiple cystic areas containing viscous liquid or a semi-solid substance are surrounding the mass anteriorly. The lesion includes rich peripheral and central hypervascular flow pattern (b). High resistance arterial flow is observed in the wall of the cyst (c) and centre (d) of the lesion. T2W Fat saturated axial image (e) shows that the mass is deeply located and invaded the rectus muscle and multiple hyperintense cysts are observed around the mass (short arrows). A $1.5 \mathrm{~cm}$ satellite nodule is seen on the left side of the primary lesion (long arrow)
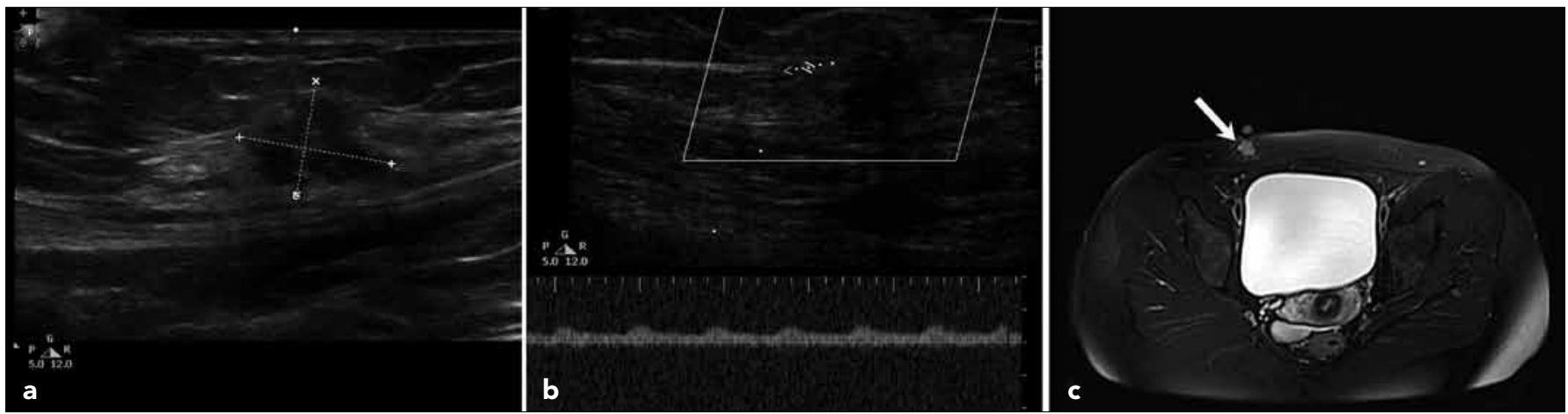

Figure 3. a-c. A 29 year-old woman develops marked right lower quadrant abdominal pain. She was referred to us with the suspicion of acute appendicitis. She had undergone a cystectomy operation on the right ovary about one year ago. Ultrasound examination (a) shows $15 \times 11 \mathrm{~mm}$ hypoechoic solid mass which may mimic a malignancy. The lesion was located in subcutaneous fat. Note the absence of blood vessels within the nodule at Doppler examination (b). T2W fat sat axial MRI (c) image shows a superficially located and well-demarcated hyperintense nodular lesion (arrow)

larity was observed in 5 nodules (moderately vascular $n=4$, highly vascular $n=1$ ) and no vascularity was seen in 3 nodules (Figure 3). MRI revealed the lesions to be isointense compared to muscle on the T1-weighted sequence, as high signal intensity on fat-saturated T2-weighted images, and with marked-homogeneously contrast enhancement (Figure 1d). There was a significant correlation between AWE sizes with repeated sections and the mean time between the last operation and admission to hospital $(p<0.05)$. Cyclic pain was more frequent in small endometriomas, whereas continuous pain or painless lumps were more often seen in patients with larger lesions $(p<0.05)$ (Table 1$)$.

\section{Discussion}

Endometriosis is a term used to depict the abnormal location of functional endometrial tissue outside the uterine endometrial cavity. Its incidence is dependent on the diagnostic method and choice of sampling framework. Approximately $11 \%$ of women have undiagnosed endometriosis at the popu- 
lation level, but it is difficult to accurately determine the prevalence (4-6). Extrapelvic endometriosis affects nearly every organ, except the heart and the spleen, but its most common location is in the abdominal wall (7-9). When endometriosis develops as a local mass, the lesion is called an endometrioma (10). The relationship between intra- and extrapelvic endometriosis is not clear. Only $26 \%$ of patients presenting with extrapelvic endometrioma also have intrapelvic endometriosis (6). In our study, there was only one patient with a history of ovarian endometrioma, which was surgically removed during a previous caesarean section.

In general, the characteristic clinical symptom of endometriosis is cyclic pain associated with menstrual periods $(8,9)$. Continuous pain or painless lumps may occur, but has generally been regarded as atypical (11), which may explain why it is clinically often recognised late, as was the case in our patients. This is even more striking in particularly large lesions. Indeed, when compared to patients with smaller endometriomas, women with a large mass showed a medical history characterised by a longer interval between both the last section operation and the onset of painful symptoms before hospital admission.

Ultrasonography is often performed as a first step in the evaluation of soft tissue masses. It can in fact confirm the presence of a lesion, even if small in size, and provides information on its size, location, margins, and internal structure. Moreover, sonography can easily differentiate solid from fluid masses, which are frequently benign or posttraumatic. With regard to solid masses, however, the method lacks specificity. With the exception of lipomas, which can be recognised more confidently, sonography is not able to distinguish benign lesions from malignant ones. The sonographic appearance of abdominal wall endometrioma can be cystic, multicystic, mixed, or solid (12). The echo pattern may or may not correlate with the menstrual period, and sonographic findings are nonspecific (13). In our study, sonographic examination revealed these masses to be solid, hypoechoic lesions in the abdominal wall and to contain internal vascularity on CDUS. Only one of the endometriomas also contained cystic areas. In larger lesions, CDUS revealed multiple vascular pedicles entering the mass from different points and an abundant central vascularisation in contrast with absent central vessels seen in smaller incisional endometriomas. Larger lesions extend deeper and feed from the rich vasculature of the rectus sheath (via deep branches of inferior and superior epigastric arteries). However, small lesions are located in hypovascular subcutaneous fat, thus their growth is limited (13-15). In the presence of a rapidly growing, painless mass and the absence of previous surgery, malignant soft tissue masses in the abdominal wall should be considered in the imaging differential diagnosis. In a study by Fleischer et al., the presence of central vascularity was a highly determinative finding of malignancy for soft tissue lesions. In contrast, the absence of intralesional vascularity seemed to be a reliable sign of benignancy (16). We found prominent central vascularity in four large nodules, while only two nodules were avascular. Therefore, we believed that it is not sufficient to use diagnostic ultrasound in the differentiation of malignant lesions.
Computerised tomography (CT) involves ionising radiation and requires an intravenous contrast agent. Furthermore, it is insufficient for specific diagnosis. AWE cannot be distinguished from invasive abdominal masses by CT $(12,14,17)$. Although, typically, MRI is suited best for defining the anatomy of the soft tissue mass and its surrounding structures, the signal characteristics and contrast enhancement pattern of AWE are not specific, and the true histological nature of the tumour or other soft-tissue masses often cannot be ascertained by imaging alone, with few exceptions (e.g., lipoma or haemorrhage) $(13,18)$. However, MRI has become a useful tool in AWE to provide a roadmap for operations to determine the location and depth of infiltration in the surrounding tissues. Busard et al. investigated the diffusion-weighted imaging (DWI) MRI features of AWE and found that DWI was useful for evaluating deep AWEs (18-20). In our study, conventional MR sequences revealed non-specific imaging findings identical to that of soft tissue malignancy.

The different imaging modalities mentioned here are nonspecific but useful in determining the extent of disease, and assist in the planning of operative resection, especially in recurrent and large lesions. FNAB has been reported to diagnose endometrioma in isolated cases $(6,11,21,22)$. Because needle tract endometriosis after amniocentesis has been reported (23), it would be advisable to include the site of aspiration in the field of operative resection. In our study, the lesions localised with US were completely excised by surgeons. To date, none of the patients in this study have had a recurrence of their AWEs.

The gold standard treatment for AWE is wide excision of the mass. If the lesion is not completely removed, it can reoccur or new lesions can form if the neighbouring parenchyma is inoculated during the operation (21-24). Thus, ultrasound examination should focus on the exact prediction of the size of the mass and assessing whether or not the AWE invades the abdominal fascia. In fact, small, superficially-located nodules are relatively easy to remove. Those lesions which have invaded the rectus sheath or are located deeper in the muscle layer will require a wider laparotomy for complete resection, and the risks and healing time will be greater $(13,21,25)$.

The limitations of our study are its retrospective nature regarding only patients with AWE and the fact that no standardised protocol was predetermined for examining the patients. This condition might have led to some lack of uniformity. Against all odds, all lesions were examined using a high-frequency linear transducer and CDUS examination was performed in all masses. Furthermore, a consensus was achieved by the authors for every lesion, by reviewing several digital documentations. This should have reduced the limitations and reinforced the whole data analysis. Finally, our study is based on a limited number of patients and should be confirmed in a larger series of patients.

In conclusion, AWE is often misdiagnosed clinically because endometriosis may occur after years of operation, the pain is often non-cyclic in nature, and there is not always a palpable tender lump. Because of the lack of specific imaging features, there is no need to use of other expensive diagnostic modalities, such as MRI and CT. The sonographic and 
CDUS features described here, along with careful correlation with clinical data and patients' history (particularly presence of previous surgery adjacent to the lesion), may considerably contribute to the correct diagnosis of endometrioma.

Ethics Committee Approval: Ethics committee approval was received from the institutional review board for this study.

Informed Consent: Written informed consent was waived because of the retrospective nature of the analysis but all patients previously permitted to the use of their medical records for the purpose of any form of clinical study.

Peer-review: Externally peer-reviewed.

Author contributions: Concept - A.S.; Design - A.S.; Supervision B.G.; Resource - S.Y.; Materials - T.Ö.S., S.Y.; Data Collection\&/or Processing - A.S.; Analysis\&/or Interpretation - I.S.; Literature Search - A.S. Writing - A.S., N.Ş. Critical Reviews - S.Y.

Conflict of Interest: No conflict of interest was declared by the authors.

Financial Disclosure: No financial disclosure was declared by the authors.

\section{References}

1. Blanco RG, Parithivel VS, Shah AK, Gumbs MA, Schein M, Gerst $\mathrm{PH}$. Abdominal wall endometriomas. The American Journal of Surgery 2003;185:596-8. [CrossRef]

2. Anand $M$, Deshmukh SD. Massive abdominal wall endometriosis masquerading as desmoid tumour. J Cutan Aesthet Surg 2011;4:141-3. [CrossRef]

3. Applebaum GD, Iwanczyk L, Balingit PB. Endometrioma of the abdominal wall masquerading as hernia. Am J Emerg Med 2004;22:621-2. [CrossRef]

4. Bachir JS, Bachir NM. Scar endometrioma: awareness and prevention. WMJ 2002;101:46-9.

5. Buck Louis GM, Hediger ML, Peterson CM, Croughan M, Sundaram R, Stanford J, et al. Incidence of endometriosis by study population and diagnostic method: the ENDO study. Fertil Steril 2011;96:360-5. [CrossRef]

6. Garwood E, Kumar A, Moes Gregory, Published Online: Wednesday, August 8, 2007 Abdominal scar endometrioma mimicking incisional hernia. http://www.hcplive.com/publications/surgicalrounds. Accessed August 8, 2007

7. lafrate $F$, Ciolina $M$, lannitti $M$, Baldassari $P$, Pichi $A$, Rengo $M$, et al. Gallbladder and muscular endometriosis: a case report. Abdom Imaging 2012;37:616-27. [CrossRef]

8. Roncoroni L, Costi R, Violi V , Nunziata R. Endometriosis on laparotomy scar. A three-case report. Arch Gynecol Obstet 2001;265:165-7. [CrossRef]

9. Ozel L, Sagiroglu J, Unal A, Unal E, Gunes P, Baskent E, et al. Abdominal wall endometriosis in the cesarean section surgi- cal scar: a potential diagnostic pitfall. J Obstet Gynaecol Res 2012;38:526-30. [CrossRef]

10. Woodward PJ, Sohaey R, Mezzetti TP. Endometriosis: RadiologicPathologic Correlation. Radiographics 2001;21:193-216.

11. Pikoulis E, Karavokiros J, Veltsista K, Diamantis T, Griniatsos J, Basios $\mathrm{N}$, et al. Abdominal scar endometriosis after caesarean section: report of five cases. West Indian Med J 2011;60:351-3.

12. Savelli L, Manuzzi L, Donato ND, Salfi N, Trivella G, Ceccaroni $M$, et al. Endometriosis of the abdominal wall: ultrasonographic and Doppler characteristics. Ultrasound Obstet Gynecol 2012;39:336-40. [CrossRef]

13. Francica G. Reliable clinical and sonographic findings in the diagnosis of abdominal wall endometriosis near cesarean section scar. World J Radiol 2012;28:135-40. [CrossRef]

14. Stein L, Elsayes KM, Wagner-Bartak N. Subcutaneous abdominal wall masses: radiological reasoning. AJR Am J Roentgenol 2012;198:146-51. [CrossRef]

15. Picard A, Varlet $M N$, Guillibert $F$, Srour $M$, Clemenson $A$, Khaddage $A$, et al. Three-dimensional sonographic diagnosis of abdominal wall endometriosis: a useful tool? Fertil Steril 2011;95:289.e1-4.

16. Fleischer AC, Milam MR, Crispens MA, Shappell HW. Sonographic depiction of intratumoral vascularity with 2- and 3-dimensional color Doppler techniques. J Ultrasound Med 2005;24:533-7.

17. Hensen JH, Van Breda Vriesman AC, Puylaert JB. Abdominal wall endometriosis: clinical presentation and imaging features with emphasis on sonography. AJR 2006;186:616-20. [CrossRef]

18. Onbas $O$, Kantarci $M$, Alper $F$, Kumtepe $Y$, Durur I, Ingec $M$, et al. Nodular endometriosis: dynamic MR imaging. Abdom Imaging 2007;32:451-6. [CrossRef]

19. Busard MP, Mijatovic V, van Kuijk C, Hompes PG, van Waesberghe JH. Appearance of abdominal wall endometriosis on MR imaging. Eur Radiol 2010;20:1267-76 [CrossRef]

20. Randriamarolahy A, Perrin H, Cucchi JM, Fuerxer F, Brunner $P$, Bruneton JN. Endometriosis following cesarean section: ultrasonography and magnetic resonance imaging. Clin Imaging 2010;34:113-5. [CrossRef]

21. Bektaş H, Bilsel Y, Sari YS, Ersöz F, Koç O, Deniz M, et al. Abdominal wall endometrioma; a 10-year experience and brief review of the literature. J Surg Res 2010;164:77-81. [CrossRef]

22. Kaunitz A, Di Sant'Agnese PA. Needle tract endometriosis: an unusual complication of amniocentesis. Obstet Gynecol 1979;54:753-5.

23. Pathan ZA, Dinesh U, Rao R. Scar endometriosis. J Cytol 2010;27:106-8. [CrossRef]

24. Kumakiri J, Kikuchi I, Kitade M, Kuroda K, Matsuoka S, Tokita S, et al. Incidence of complications during gynecologic laparoscopic surgery in patients after previous laparotomy. J Minim Invasive Gynecol 2010;17:480-6. [CrossRef]

25. Sawazaki H, Goto H, Takao N, Taki Y, Takeuchi H. Clear cell adenocarcinoma arising from abdominal wall endometriosis mimicking urachal tumor. Urology 2012;79:84-5. [CrossRef] 